\title{
Possible Vs. Scored Points - Statistical Analysis of LEED 2009-NCv3 Projects in Egypt
}

\author{
Dr. Mikhael Maged G. N \\ (Author) \\ Department of Architectural engineering, \\ Canadian International College, \\ Sheikh Zayed, 6th. Of October City, Egypt
}

\begin{abstract}
The number of Leadership in Energy and Environmental Design (LEED) projects in Egypt has increased since 2010. This paper aims to reveal the ambiguity about attributes, trends, and levels of achievements in these projects by conducting a statistical analysis of the LEED-awarded projects. The descriptive statistics clarified that most awarded/registered projects have applied for building design and construction certification (LEED 2009-NCv3). The author merged both dotplot and boxplot techniques to visualize and measure the achieved points in the seven categories of the mentioned version. For inferential statistics, the effect size was measured between possible and scored points in both categories and credits using the nonparametric Cliff's $\delta$ effect size test. The statistical analysis shows that: Regional Priority category had a small Cliff's $\delta$ effect size $(0.3)$, with almost half of its credits achieved high scores. Water efficiency and Innovation in design categories had a large Cliff's $\delta$ effect size (0.7), but $1 / 2$ and $2 / 3$ of their credits (respectively) achieved high scores. Sustainable Sites, Energy and Atmosphere, Materials \& Resources, and Indoor Environmental Quality had the maximum Cliff's $\delta$ effect size (1), with half (or more) of their credits achieved low-high scores.
\end{abstract}

Keywords- LEED 2009-NCv3, Egyptian LEED projects, LEED statistics, effect size

\section{INTRODUCTION}

Global warming and climate change have become unequivocal phenomena. The Intergovernmental Panel on Climate Change (IPCC) that constitutes the body of the United Nations for assessing the science related to climate changes had addressed in its Global Warming of $1.5 \mathrm{C}^{\circ}$ report in 2019: "There is an increase in the global mean surface temperature (GMST) of $0.87 \mathrm{C}^{\circ}$ in 2006-2015 compared to the preindustrial period 1850-1900". This temperature increase has many harmful effects on a range of both natural and human systems [1]. Fossil fuels generate almost $75 \%$ of electrical power in the United States, while residential and commercial buildings consume nearly $40 \%$ of this energy [2]. In Egypt, fossil fuels generate more than $90 \%$ of the electrical power, and the total consumption of both residential and commercial buildings jumps to $52.5 \%$ [3]. Since the burning of fossil fuels is one of the significant causes of greenhouse gas (GHG) hence climate change - buildings are considered one of the main reasons for the problem. This fact motivated many national and global organizations to create green building standards, certifications, and rating systems - as an endeavor to reduce the harmful effects of buildings on the natural environment through sustainable design. The Building Research Establishment's Environmental Assessment Method
(BREEM) was launched in the United Kingdom in 1990 as the first green building rating system (RS) in the world. Later, other responses appeared worldwide, such as L'Haute Qualité Environnementale (HQE) in France-1994, Leadership in Energy and Environmental Design (LEED) in the US-1998, Deutsche Gesellschaft für Nachhaltigies Bauen (DGNB) in Germany-2009 and others. Among the so many different rating systems, LEED and BREEM are the most popular internationally [4]. Also, the Housing and Building National Research Center (HBNRC) in Egypt introduced the Green Pyramid rating system (GPRS) in 2009 [5]. Taking the v3LEED 2009 as a starting point to build on, the (HBNRC) published the newest version of the GPRS (V2) in 2017 [6]. Although GPRS is eleven years old now, it is not applied to a wide range in the Egyptian construction market due to many legislative and educational reasons. Meanwhile, LEED is one of the most recognized rating systems in Egypt [7]. The number of LEED awarded/registered projects in Egypt is increasing since the HSBC bank processing center in Cairo had been awarded - in 2010-the Gold certificate as the first LEEDawarded project in Egypt and Africa. During the last decade (January 2010 - November 2020), twenty-one projects had been awarded LEED certificates on different certification levels, while thirty-six projects had already registered in six governorates (Figure-1). Figure -2 shows that nearly half the number of LEED-awarded projects in Egypt - Ten out of the twenty-one - were certified according to the building design and construction for new constructions - LEED BD+C: New construction (v2009) - version (LEED 2009-NCv3). Furthermore, almost a third of the registered projects - eleven out of thirty-six - had already applied to the same version [8].

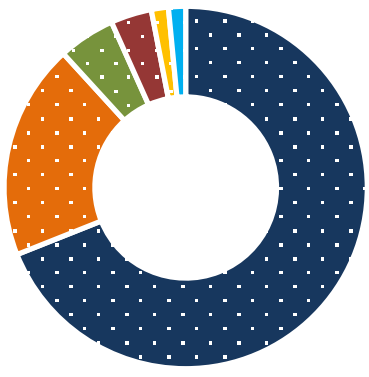

$$
\begin{aligned}
& \text { - Cairo 39/57 } \text { " Giza 11/57 } \\
& \text { - Alexandria 3/57 } \text { " Minya 2/57 } \\
& \text { " Suez 1/57 } \quad \text { " Minufeya 1/57 }
\end{aligned}
$$

Fig. 1. Distribution of LEED-awarded projects on six governorates in Egypt 


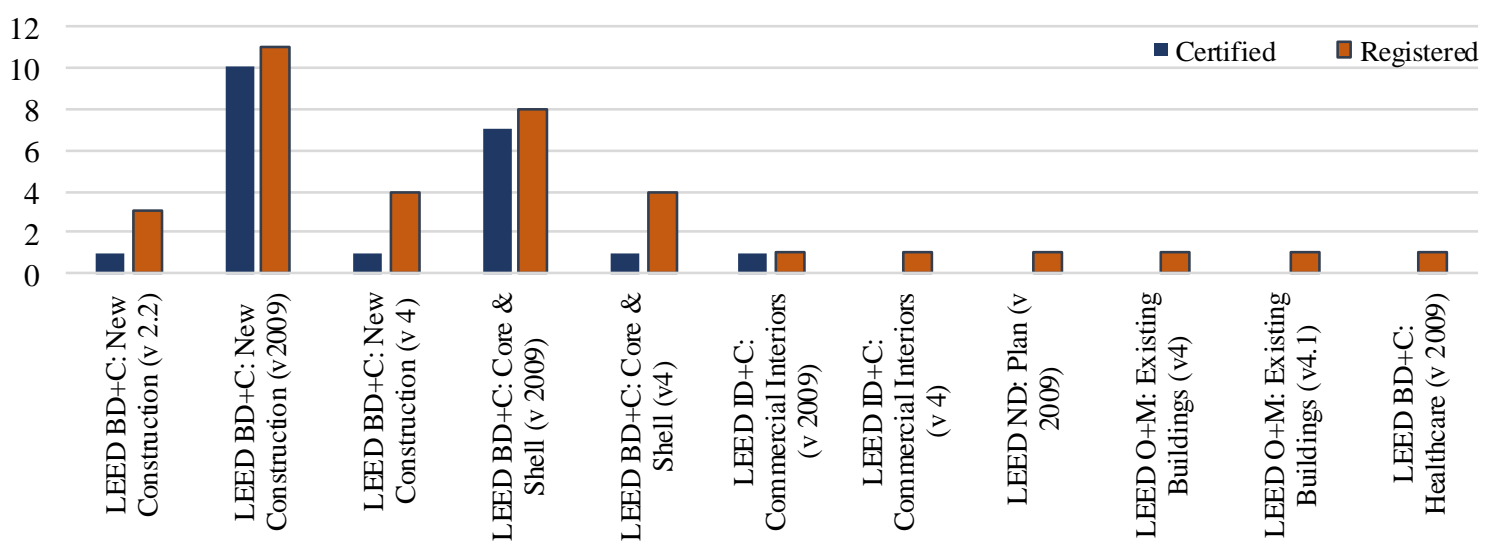

Fig. 2. Count of registered/awarded projects in Egypt according to different LEED versions.

The current version of LEED 2009-NCv3 is composed of seven environmental categories with 110 points in total. The segmentation of total points is as follows: 26 points for the Sustainable Sites (SS), 10 points for Water Efficiency (WE), 35 points for Energy \& Atmosphere (EA), 14 points for Material \& Resources (MR), 15 points for Indoor Environmental Quality (EQ), 6 points for Innovation (ID) and 4 points for Regional Priority (RP). Based on the total achieved points, a project can earn one of the four green buildings certificates: certified (40-49 points), silver (50-59 points), Gold (60-79 points), or platinum (80 or more points) [9]. Many types of research covered the different rating systems - Including the GPRS - in the Egyptian construction industry since 2012. Some researchers focused on evaluating or comparing the different rating systems [10, $11 \& 12]$. Others were interested in the assessment of contemporary or historical buildings according to the rating systems [13 \& 14]. A third party studied the applicability and marketing of the different rating systems $[6,7,15,16 \& 17]$. While a little focused on the cost and energy perspective [18]. From the previous literature, it is evident that:

- There is an increasing interest - and awareness between the Egyptian developers and designers towards introducing LEED-certified buildings.

- The last research pieces did not include any statistical analysis that may clarify the most popular LEED version(s) in the Egyptian market or the levels of achievements for the environmental categories/credits in this/these version(s).

\section{RESEARCH OBJECTIVES AND QUESTIONS}

The main objectives of this research are to:

- understand the common attributes that characterize the LEED-awarded/registered projects in Egypt and classify the LEED 2009-NCv3 environmental categories/credits according to their certified Egyptian construction projects' achievements.

- Utilizing the previous classification to guide both decision-makers \& designers during the pre-design and design stages.
The previous objectives were the influencing force behind formulating the following research questions:

- What are the main attributes and trends of the LEEDawarded/registered projects in Egypt?

- Are there any environmental categories/credits - in the LEED 2009-NCv3 - that have higher achievement potential in the Egyptian construction market?

- Can the previous question's answer be utilized to guide both decision-makers and designers during the predesign/design stages?

\section{METHODOLGY}

Since this research intends to find answers for open-end questions rather than examining a pre-determined theory/hypothesis, the quantitative research strategy will be the most appropriate choice for this target [19]. Thus, this research will pass through the following two main phases:

\section{A. Phase One (Drilling in)}

This phase includes Data collection, reduction \& organization.

- Data collection: As the main target of this research is to examine the LEED-awarded projects in Egypt, it was crucial to refer to the official website of the U.S. Green Building Council (USGBC) and check its directory for the Egyptian projects. Twenty-one soft copies of the certified scorecards are available in the directory; these scorecards include all the essential raw data to conduct this research [8].

- Data reduction and organization: Among the twentyone awarded projects, eighteen were assessed according to LEED 2009-NCv3 as follows: seven for Core \& Shell (C\&S), one for Commercial Interiors (CI), and ten for New Construction (NC). Only the scorecards of the (NC) projects will constitute the needed data for this research. The author did not compare the different certification levels in this study since half of the projects (five) earned the silver certificate, while three earned the gold, one earned the platinum certificate, and only one is certified. Finally, it was necessary to extract and tabulate the different scores of each environmental category/credit before moving to the statistical analysis. 


\section{B. Phase Two (Abstracting out phase)}

This phase is dedicated to carrying out the statistical analysis, presenting the thematic data, and the meaningful theoretical understanding of findings; thus, it is structured as follows:

- Descriptive statistics: Since the number of projects (variables) is not more than ten, the author preferred to use a mix of dot-plot and box-plot techniques to illustrate the descriptive statistics for different environmental categories. Simultaneously, the medianinterquartile range (IQR, 25th. - 75th. percentile) method is better for evaluating the LEED 2009-NCv3 credits than the mean \pm standard deviation since LEED data are ordinal [20]. The author used the interactive dot plot online tool - a free tool for interactive graphics - to generate the dot-plot/box-plot charts and calculate the median (the line inside the box), the 25th. \& 75th. percentiles of IQR represent the upper and lower sides of the box, while the whiskers extend to the points that are extremisms but not outliers [21].

- Statistical analysis: To get inferential statistics considering the data nature of LEED - S. Pushkar 2020, in "Evaluating State-of-the-Art LEED-NCv4 in the U.S.", used the nonparametric Cliff's $\delta$ effect size test to measure the difference magnitude between two different distributions - possible (group 1) and achieved points (group 2) in categories/credits - as it does not depend on the sample size. This approach is used in this study as well since the data type is similar [22]. Cliff's $\delta$ effect size ranges between 1 and -1 ; its positive values point out that group 1 is larger than 2 . Zero value points out an overlap between the two groups, while negative values point out that group 2 is larger than 1 [23]. When the $\mid$ Cliff's $\delta \mid$ is $<0.147$ it is negligible, it is small if $0.147<\mid$ Cliff's $\delta \mid<0.33$, it is medium if $0.33<\mid$ Cliff's $\delta \mid<0.474$ and it is large if $\mid$ Cliff's $\delta \mid \geq 0.474$ [24]. It was necessary to consider both the median and range of data distribution to evaluate each category's consistency in the scored points. Hence, a wide range indicates an inconsistency in scored points in one category, while a narrow range indicates consistency in scored points in another one. Also, to evaluate Cliff's $\delta$ effect sizes between possible and scored points, the reformulation of effect size that was suggested by Pushkar 2020 will be applied. "Very high achieved points if $|\delta|<0.147$, high achieved points if $0.147<|\delta|<0.33$, medium achieved points if $0.33<|\delta|<0.474$, and low achieved points if $|\delta| \geq$ 0.474. " [22].

\section{STATISTICAL RESULTS}

\section{A. Sustainable Sites (SS):}

The boxplot (Figure 3) shows a wide range for the scored points (9-22) in the SS category accompanied by a median of 17 and a large Cliff's $\delta$ effect size of (1) while the projects' scores show a relatively normal distribution. Table 1 shows the possible points in addition to the calculation results of median \pm IQR and Cliff's $\delta$ effect size between the possible and scored points for all the SS credits. Five credits in the SS

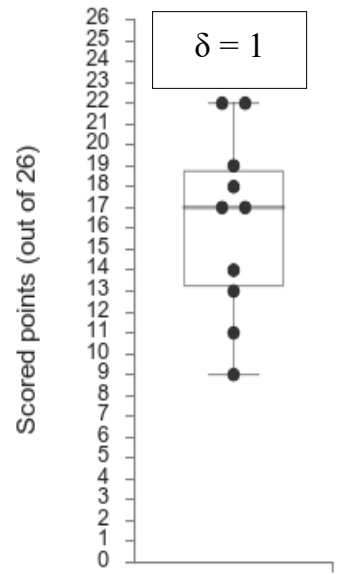

Fig. 3 Boxplot/Dot plot \& Cliff’s $\delta$ results for SS category

Table 1. CREDITS' POSSIBLE POINTS, FOR (SS) CATEGORY: MEDIAN \pm IQR \& CLIFF'S $\delta$ EFFECT SIZE BETWEEN POSSIBLE AND SCORED POINTS IN EACH CREDIT.

\begin{tabular}{|c|c|c|c|}
\hline Credit & $\begin{array}{c}\text { Possible } \\
\text { points }\end{array}$ & $\begin{array}{c}\text { Median } \\
\pm \\
\text { IQR }\end{array}$ & Cliff's $\delta$ \\
\hline SSc1 & 1 & $1 \pm 0$ & 0 \\
\hline $\mathrm{SSc} 2$ & 5 & $0 \pm 0$ & 0.8 \\
\hline SSc3 & 1 & $0 \pm 0$ & 1 \\
\hline SSc4.1 & 6 & $6 \pm 0$ & 0.2 \\
\hline $\mathrm{SSc} 4.2$ & 1 & $1 \pm 0$ & 0.1 \\
\hline $\mathrm{SSc} 4.3$ & 3 & $3 \pm 0$ & 0 \\
\hline $\mathrm{SSc} 4.4$ & 2 & $2 \pm 0$ & 0.2 \\
\hline SSc5.1 & 1 & $0 \pm 1$ & 0.6 \\
\hline SSc5.2 & 1 & $1 \pm 1$ & 0.4 \\
\hline SSc6.1 & 1 & $0 \pm 1$ & 0.6 \\
\hline SSc6.2 & 1 & $0 \pm 0.75$ & 0.7 \\
\hline SSc7.1 & 1 & $1 \pm 1$ & 0 \\
\hline SSc7.2 & 1 & $1 \pm 1$ & 0 \\
\hline SSc8 & 1 & $0 \pm 0$ & 0.8 \\
\hline Total & 26 & & \\
\hline
\end{tabular}

category had a negligible Cliff's $\delta$ effect size. Site selection (SSc1), alternative transportation - bicycle storage and changing rooms (SSc4.2), alternative transportation - lowemitting and fuel-efficient vehicles (SSc4.3), Heat island effect - nonroof (SSc7.1) and Heat island effect-roof (SSc7.2) had achieved the maximum score (1 point) in all the projects. Alternative transportation - public transportation access and Alternative transportation - parking capacity (SSc4.1 and SSc4.4 respectively) had a small Cliff's $\delta$ effect size (0.2) as eight of the projects achieved the maximum score of these credits. Site development - maximize open space (SSc5.2) had a medium Cliff's $\delta$ effect size; hence medium achieved points. While, development density and community connectivity (SSc2), brownfield redevelopment (SSc3), site development protect or restore habitat (SS5.1), Storm-water design quantity control (SSc6.1), Storm-water design - quality control (SSc6.2), and Light pollution reduction (SSc8) had large Cliff's $\delta$ effect size $\geq 6$.

\section{B. Water Efficiency (WE):}

As illustrated in figure 4, a wide range of total scored points is apparent (4-10), but it is noticeable that the median is 
relatively high (8), and most of the projects already achieved high scores (seven projects achieved $\geq 7$ points) with a large Cliff's $\delta$ effect size (0.7). Table 2 summarizes the median \pm IQR (25th. -75 th.) and Cliff's $\delta$ effect size between the possible and scored points for each WE credit. Water- efficiency landscaping (WEc1) and water use reduction (WEc3) have a small Cliff's $\delta$ effect size $(0.2 \&$ o.3), respectively. These values clarify that most of the projects could reduce or eliminate the consumption of potable water and the natural surface water or groundwater for landscape irrigation to the minimum and increase the water efficiency usage within the buildings. While the Innovative wastewater technologies credit (WEc2) has a large Cliff's $\delta$ effect size (0.7) as most of the projects failed to incorporate innovations that can minimize the generation of wastewater and reduce the potable water need.

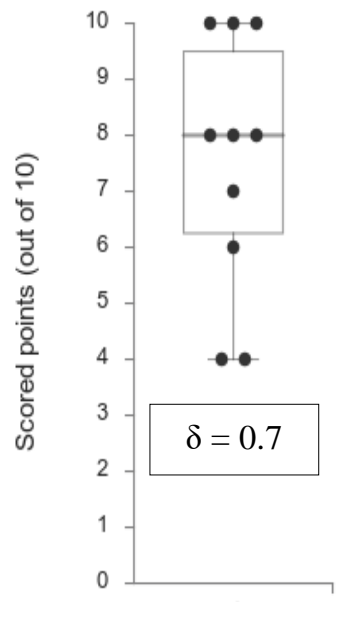

Fig. 4 Boxplot/Dot plot \& Cliff’s $\delta$ results for WE category

Table 2. CREDITS' POSSIBLE POINTS, FOR (WE) CATEGORY: MEDIAN \pm IQR \& CLIFF'S $\delta$ EFFECT SIZE BETWEEN POSSIBLE AND SCORED POINTS IN EACH CREDIT.

\begin{tabular}{|l|c|c|c|}
\hline Credit & $\begin{array}{c}\text { Possible } \\
\text { points }\end{array}$ & $\begin{array}{c}\text { Median } \\
\mathbf{\pm} \\
\text { IQR }\end{array}$ & Cliff's $\boldsymbol{\delta}$ \\
\hline WEc1 & 4 & $\mathbf{4} \pm \mathbf{0}$ & $\mathbf{0 . 2}$ \\
\hline WEc2 & 2 & $\mathbf{0} \pm \mathbf{1 . 5}$ & $\mathbf{0 . 7}$ \\
\hline WEc3 & 4 & $\mathbf{4 \pm 0 . 7 5}$ & $\mathbf{0 . 3}$ \\
\hline Total & 10 & \multicolumn{2}{|l}{} \\
\cline { 1 - 2 }
\end{tabular}

\section{Energy and Atmosphere (EA):}

In the EA category, most of the projects achieved relatively low scores (median is 11.5) and a large Cliff's $\delta$ effect size (1). However, the range is vast (2-26) due to the high score - an upper outlier - of only one project (figure 5). Table 3 shows the median \pm IQR and Cliff's $\delta$ effect size between the possible and scored points for all EA credits. Four of the six credits in the category have a large Cliff's $\delta$ effect size between the possible and scored points. Optimize energy performance (EAc1), onsite renewable energy (EAc2), enhanced commissioning (EAc3), and green power (EAc7) credits have either zero or relatively low scores in most of the projects. Simultaneously, the enhanced refrigeration management (EAc4) and the measurement and verification (EAc5) had a negligible and small Cliff's $\delta$ effect size, respectively, and achieved the maximum score in eight and nine of the projects, respectively.

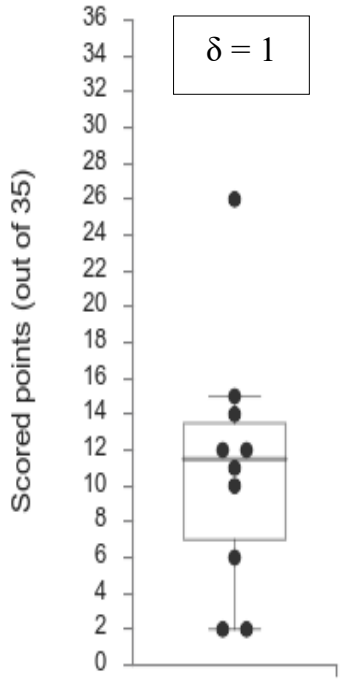

Fig. 5 Boxplot/Dot plot \& Cliff's $\delta$ results for EA category

TABle 3. CREDITS' POSSIBLE POINTS, FOR (EA) CATEGORY: MEDIAN \pm IQR \& CLIFF'S $\delta$ EFFECT SIZE BETWEEN POSSIBLE AND SCORED POINTS IN EACH CREDIT.

\begin{tabular}{|l|c|c|c|}
\hline Credit & $\begin{array}{c}\text { Possible } \\
\text { points }\end{array}$ & $\begin{array}{c}\text { Median } \\
\pm \\
\text { IQR }\end{array}$ & Cliff's $\boldsymbol{\delta}$ \\
\hline EA c1 & 19 & $4 \pm 5$ & 0.9 \\
\hline EAc2 & 7 & $0 \pm 2$ & 1 \\
\hline EAc3 & 2 & $0 \pm 2$ & 0.8 \\
\hline EAc4 & 2 & $2 \pm 0$ & 0.1 \\
\hline EAc5 & 3 & $3 \pm 1.5$ & 0.3 \\
\hline EAc6 & 2 & $0 \pm 1.5$ & 0.7 \\
\hline Total & 35 & \multicolumn{2}{l}{} \\
\cline { 1 - 3 }
\end{tabular}

\section{Materials \& Resources (MR):}

As shown in figure 6 , the range of total scored points in the MR is relatively wide (2-7) while the median is low (4.5), and the Cliff's $\delta$ effect size is large (1). This depicts that the scored points for this category were low for all the projects. The median \pm IQR and Cliff's $\delta$ effect size between the possible and scored points for all MR credits are illustrated in table 4 . Building reuse-maintain existing walls, floors, and roof (MRc1.1) Building reuse - maintain interior nonstructural elements (MRc1.2), Materials reuse (MRc3), and Rapidly renewable materials (MRc6) have a large Cliff's $\delta$ effect size (1) hence, none of the projects scored any points for any of these credits. Since all the projects are new constructions in new urban communities, the (MRc1.1 \& MRc1.2) were not applicable. Also, reusing building material or depending on renewable construction materials and products that are made from rapidly harvested agricultural products (MRc3 and MRc6, respectively) are still not standard practices in the modern Egyptian construction industry. Construction waste management (MRc2), Recycled content (MRc4), and certified wood (MRc7) credit had large Cliff's $\delta$ effect size as well, hence low achievement. The regional materials credit (MRc5) is the only one in this category that had negligible Cliff's $\delta$ effect size and achieved a high score. 


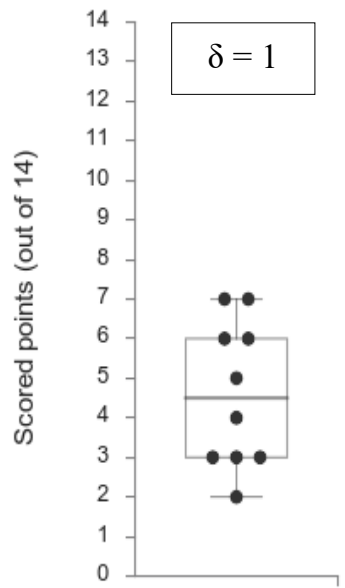

Fig. 6 Boxplot/Dot plot \& Cliff's $\delta$ results for MR category

TABLE 4. CREDITS' POSSIBLE POINTS, FOR (MR) CATEGORY: MEDIAN \pm IQR \& CLIFF'S $\delta$ EFFECT SIZE BETWEEN POSSIBLE AND SCORED POINTS IN EACH CREDIT.

\begin{tabular}{|l|c|c|c|}
\hline Credit & $\begin{array}{c}\text { Possible } \\
\text { points }\end{array}$ & $\begin{array}{c}\text { Median } \\
\pm \\
\text { IQR }\end{array}$ & Cliff's $\boldsymbol{\delta}$ \\
\hline MRc1.1 & 3 & $0 \pm 0$ & 1 \\
\hline MRc1.2 & 1 & $0 \pm 0$ & 1 \\
\hline MRc2 & 2 & $1.5 \pm 1.75$ & 0.5 \\
\hline MRc3 & 2 & $0 \pm 0$ & 1 \\
\hline MRc4 & 2 & $1 \pm 1$ & 0.6 \\
\hline MRc5 & 2 & $2 \pm 0$ & 0.1 \\
\hline MRc6 & 1 & $0 \pm 0$ & 1 \\
\hline MRc7 & 1 & $0 \pm 0.75$ & 0.7 \\
\hline Total & 14 & &
\end{tabular}

\section{E. Indoor Environmental Quality (EQ):}

Figure 7 shows the boxplot results in the EQ category. As illustrated, the range of the scored points is vast; the median has an average value (9) while the distribution of the projects' scored points is close to normal. Table 5 provides the median \pm IQR and Cliff's $\delta$ effect size between the possible and scored points for $\mathrm{EQ}$ credits. Increased ventilation (EQc2), Construction IAQ management plan - during construction (EQc3.1), and Low-emitting materials - paints and coatings (EQc4.2) had negligible Cliff's $\delta$ effect size, which means that these credits had very high achievement. Outdoor air delivery monitoring (EQc1), Low-emitting materials - adhesives and sealants (EQc4.1), Thermal comfort - design (EQc7.1), and thermal comfort - verification (EQc7.2) had small Cliff's $\delta$ effect size indicating that these credits were achievable in most of the projects (seven or more). Construction IAQ management plan - before occupancy (EQc3.2), Low-emitting materials-flooring systems (EQc4.3), Low-emitting materials composite wood and agrifiber products (EQc4.4), indoor chemical and pollutant source control (EQc5), Controllability of systems - lighting (EQc6.1), Controllability of systems thermal comfort (EQc6.2), Daylight and views dayligh(EQc8.1), and Daylight and views - views (EQc8.2) had large Cliff's $\delta$ effect size as only 2-5 projects achieved these credits.

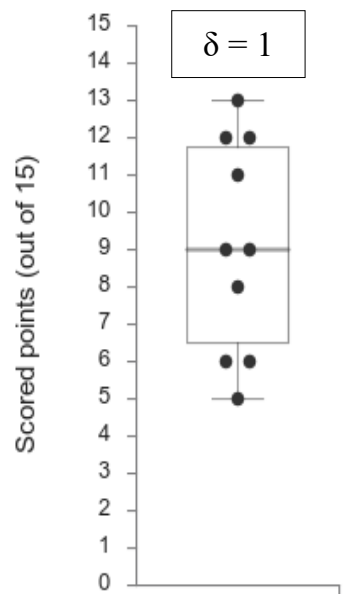

Fig. 7 Boxplot/Dot plot \& Cliff’s $\delta$ results for EQ category

TABLE 5. CREDITS' POSSIBLE POINTS, FOR (EQ) CATEGORY: MEDIAN \pm IQR \& CLIFF'S $\delta$ EFFECT SIZE BETWEEN POSSIBLE AND SCORED POINTS IN EACH CREDIT.

\begin{tabular}{|l|c|c|c|}
\hline Credit & $\begin{array}{c}\text { Possible } \\
\text { points }\end{array}$ & $\begin{array}{c}\text { Median } \\
\pm \\
\text { IQR }\end{array}$ & Cliff's $\boldsymbol{~}$ \\
\hline EQc1 & 1 & $1 \pm 1$ & 0.3 \\
\hline EQc2 & 1 & $1 \pm 0$ & 0.1 \\
\hline EQc3.1 & 1 & $1 \pm 0$ & 0 \\
\hline EQc3.2 & 1 & $0 \pm 0.75$ & 0.7 \\
\hline EQc4.1 & 1 & $1 \pm 0.75$ & 0.3 \\
\hline EQc4.2 & 1 & $1 \pm 0$ & 0 \\
\hline EQc4.3 & 1 & $0 \pm 1$ & 0.6 \\
\hline EQc4.4 & 1 & $0 \pm 1$ & 0.6 \\
\hline EQc5 & 1 & $1 \pm 1$ & 0.4 \\
\hline EQc6.1 & 1 & $0 \pm 1$ & 0.5 \\
\hline EQc6.2 & 1 & $0 \pm 1$ & 0.6 \\
\hline EQc7.1 & 1 & $1 \pm 0$ & 0.2 \\
\hline EQc7.2 & 1 & $1 \pm 0$ & 0.2 \\
\hline EQc8.1 & 1 & $0 \pm 0$ & 0.8 \\
\hline EQc8.2 & 1 & $0 \pm 0.75$ & 0.6 \\
\hline Total & 15 & \multicolumn{2}{|l}{} \\
\cline { 1 - 3 } & \multicolumn{2}{|l}{} &
\end{tabular}

\section{F. Innovation in design (ID):}

Although the ID category shows a relatively wide range of scored points (2-6), the median is very high (5), and most of the projects ( 9 projects) accomplished a high or the maximum score (Figure 8). In table 6, it is evident that one credit (IDc2) of the ID category had negligible Cliff's $\delta$ effect size (0) between the possible and scored points, which means that at least one LEED-Accredited Professional (AP) worked as a principal participant in the team of each project. At the same time, the other credit (IDc1) had a large Cliff's $\delta$ effect size (o.7) due to the varying values of scored points in this credit.

\section{G. Regional Priority $(R P)$ :}

This category is the only one that has a small range of scored points (3-4) and a very high median (4) due to that most of the projects ( 7 projects) achieved the maximum score for this category, while the rest ( 3 projects) achieved 3 points out of 4 (figure 9). The RP category has the smallest Cliff's $\delta$ effect size (o.3) among all the eight environmental categories. The calculations of the Median \pm IQR \& Cliff's $\delta$ effect size between possible and scored points in each credit is 
meaningless for this category as it includes four bonus points that may result from performing regional related credits in SS, WE, EA, and MR.

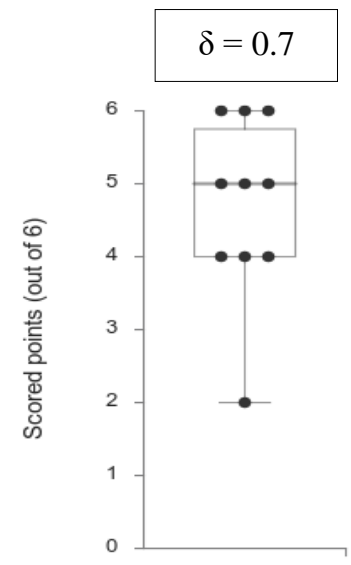

Fig. 8 Boxplot/Dot plot \& Cliff's $\delta$ results for ID category

TABLE 6 - CREDITS' POSSIBLE POINTS, FOR (ID) CATEGORY: MEDIAN \pm IQR \& CLIFF'S $\delta$ EFFECT SIZE BETWEEN POSSIBLE AND SCORED POINTS IN EACH CREDIT.

\begin{tabular}{|l|c|c|c|}
\hline Credit & $\begin{array}{c}\text { Possible } \\
\text { points }\end{array}$ & $\begin{array}{c}\text { Median } \\
\mathbf{\pm} \\
\text { IQR }\end{array}$ & Cliff's $\boldsymbol{\delta}$ \\
\hline IDc1 & 5 & $4 \pm 1.75$ & 0.7 \\
\hline IDc2 & 1 & $1 \pm 0$ & 0 \\
\hline Total & 6 & \multicolumn{2}{|l}{} \\
\cline { 1 - 2 }
\end{tabular}

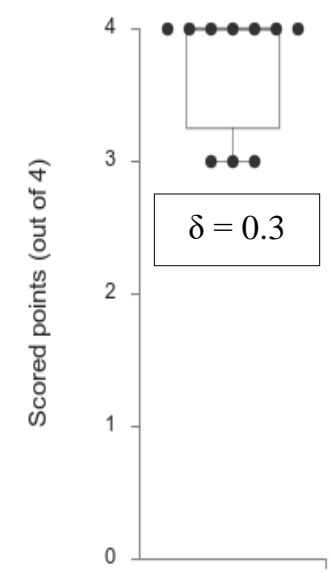

Fig. 9 Boxplot/Dot plot \& Cliff's $\delta$ results for RP category

\section{DISCUSSION}

The statistical analysis results revealed that six of the seven environmental categories of LEED 2009-NCv3 have a wide range of scored points, and only the RP category has a small range. This study also declared that the WE, ID, and RP categories were the highest-achieved categories. Although SS
\& EQ categories have a small achievement in the total score, almost half of the credits in both have very high, high, or medium achievements. EA \& MR were the worst in the seven categories as most of their credits have achieved low scores. Regarding the EA category, most of the projects did not give big attention to optimizing the energy performance (EAc1), on-site renewable energy (EAc2), Enhanced commissioning (EAc3), and the green power (EAc6) credits since these trends are hardly trying to find its rightful place in the Egyptian construction market due to cultural and economic reasons. At the same time, seven of the eight credits in MR category which are related to the reuse of buildings' elements, material recycling or reuse, management of waste, or using certified wood and rapidly renewable materials have low achievement. Moreover, despite that, WE \& ID categories the Innovative wastewater technologies (WEc2) and Innovation in Design (IDc1) credits achieved low scores thus, LEED failed to encourage the innovation in the construction industry. Similar study should be carried out to analyze the core \& shell (C \& S) LEED-awarded projects according to the same version and compare the results in both studies.

\section{CONCLUSION}

This paper presented overview research to the Egyptian LEED-awarded projects from January 2010 till November 2020 and conducted a statistical analysis of ten LEED 2009$\mathrm{NCv} 3$ projects. The following conclusions are drawn:

- The awareness of constructing green buildings is still centralized in/around Cairo the great (50 out of 57 projects), although some individual trials started in other governorates.

- Twenty-one of the fifty-seven projects either certified or registered to LEED 2009-NCv3. Simultaneously, fifteen projects were either certified or registered for the same version but core \& shell (LEED 2009C\&Sv3).

- WE, ID, and RP categories have the highest possible achievements in the Egyptian construction market, thus necessitating these credits to take the most significant concern from owners, developers, and designers who seek high certification levels. At the same time, designers should give more attention to innovative elements.

- $\quad$ SS \& EQ categories have the potential of achieving reasonable scores through many of their rewarding credits. These credits should take priority during the early preliminary design phases.

- This study found that EA \& ID have the lowest achieved scores in most of the projects. Nevertheless, designers who target higher certification levels should not discard these categories and must incorporate renewable and green energy sources in their designs. Meanwhile, they should try to apply the principle of reusing existing buildings' elements and using recycled and fast renewable materials on a larger scale. 


\section{REFRENCES}

[1] V. Masson-Delmotte, et.al, Global Warming of $1.5^{\circ} \mathrm{C}$, IPCC (2019), pp. 175-181, available online: https://www.ipcc.ch/site/assets/uploads/sites/2/2019/06/SR15_Chapter3 _Low_Res.pdf (accessed 1 November 2020).

[2] USEIA. U.S. Energy Information Administration. Use of energy explained. Available online: https://www.eia.gov/energyexplained/useof-energy/ (accessed on 1 November 2020)

[3] MOEE. Egyptian Ministry of Electricity \& Energy. Egyptian electricity holding company annual report 2018/2019. Available online: http://www.moee.gov.eg/english_new/EEHC_Rep/2018-2019en.pdf (accessed 1 November 2020)

[4] A. Cordero, S. Melgar and J. Marqueze, Green Building Rating Systems and the New Framework Level(s): A Critical Review of Sustainability Certification within Europe, Energies, 2020, vol. 13, 66. doi:10.3390/en13010066

[5] EGBC. Egyptian Green Building Council. Brief History of the Egyptian Green Building Council, Available on line: http://www.egyptgbc.org/history.html , 2020 (accessed on 5 November 2020)

[6] W. Ismaeel, A. Rashed and E. Toulaibah, To be or not to be: The national green pyramid rating system. Green Heritage Conference. Elain Publishing Company, 2018, pp. L- LXII

[7] R. Moussa, The reasons for not implementing Green Pyramid Rating System in Egyptian buildings, Ain Shams Engineering Journal, 10, vol. 4, 2019, pp. 917-927.

[8] USGBC. US Green Building Council (USGBC). Projects Directory. Available https://www.usgbc.org/projects?Country=\%5B $\% 22$ Egypt $\% 22 \% 5 \mathrm{D}$ (accessed on 10 November 2020)

[9] LEED. LEED v3 for New Construction and Major Renovations. 2009. Available online: https://www.usgbc.org/resources/leed-newconstruction-v2009-current-version (accessed on 20 November 2020)

[10] G. Ammar, Evaluation of the Green Egyptian Pyramid, Alexandria Engineering Journal, 51, vol. 4, 2012, pp. 293-304.

[11] H. Karamany, Evaluation of Green Building Rating Systems for Egypt, The American University in Cairo, Egypt, 2016.

[12] E. Attiya, M. Shebl and M. Nasser, A Comparative Analysis of LEED and GPRS for the Applicability in Egyptian Office Buildings, International Journal of Engineering Research \& Technology (IJERT), 9, 3, 2020, pp. 257-269.

[13] W. Ali and N, Mohamed, Green Architecture Assessment System in Egypt with an Application on Zeinab Khatoun House, The Internationa Institute for Science, Technology and Education (IISTE), 3, vol. 14 ,2013, pp. 56-78.

[14] N. Alkhozondar, Evaluation of the Arab Academy for Science, Technology \& Maritime Transport (AASTMT) Building to be Oriented towards a Green Building, AASTMT, College of Engineering and Technology (Cairo), 2015

[15] K. Ayyad, Markeeting Green Architecture in Egypt: Integration of Green Architecture into the Mainstream Construction Market in Egypt, Faculty of Engineering at Cairo University, 2012

[16] S. El Yamany, Applicability and Implementation of U.S. Green Building Council Rating System (LEED) in Egypt. Case Studies: LEED certified $\&$ Registered Buildings in Egypt, Faculty of Engineering at Cairo University, Egypt, 2013.

[17] W. Ali, An Applicable Approach to Green Architecture in Egypt: Proposed Measurement Matrix Model to Assess the use of Historic Islamic Architectural Elements and Repertoire in the Application of Green Architecture Principles and Concepts, Global Journal of Arts Humanities and Social Sciences, 2, vol. 10, 2014,PP. 6-29.

[18] M. Assad, O. Hosny and A. elhakeem, Green building design in Egypt from cost and energy perspectives, Architectural Engineering and Design Management, 11, vol. 1, 2013.

[19] L. Groat and D. Wang, Architectural Research Methods, 2nd. Ed., John Wiley \& Sons, New York, 2013, pp. 243-257.

[20] M.J. Campbell and T. D. V. Swinscow., Statistics at Square One, 11th. Ed. Wiley-Blackwell, 2009, pp. 1-11.
[21] T. Weissgerber et al, Data visualization, bar naked: A free tool for creating interactive graphics. Journal of Biological Chemistry, 292(50) (2017), 20592-20598. http://10.1074/jbc.RA117.000147

[22] S. Pushkar, Evaluating State-of-the-Art LEED-NCv4 in the U.S., Applied Sciences, 2020, 10(3):775.

[23] N. Cliff, Dominance statistics: Ordinal analyses to answer ordinal questions. Psychol. Bull. 1993, 114, PP. 494-509.

[24] J. Romano, J. Corragio, and J. Skowronek, Appropriate statistics for ordinal level data: Should we really be using t-test and Cohen's d for evaluating group differences on the NSSE and other surveys? In Proceedings of the Annual Meeting of the Florida Association of Institutional Research, Cocoa Beach, FL, USA, 1-3 February 2006; Florida Association for Institutional Research: Cocoa Beach, FL, USA, 2006, pp.1-33. 